\title{
Advanced Temporal Dilated Convolutional Neural Network for a Robust Car Driver Identification
}

\author{
Francesco Rundo, Francesca Trenta, Roberto Leotta, Concetto Spampinato, \\ Vincenzo Piuri, Sabrina Conoci, Ruggero Donita Labati, Fabio Scotti, and \\ Sebastiano Battiato \\ 1 STMicroelectronics, ADG Central R\&D \\ ${ }^{2}$ University of Catania, IPLAB Group \\ ${ }^{3}$ University of Catania, PerCeiVe Lab \\ 4 University of Messina, \\ 5 University of Milan, Computer Science Department \\ francesco.rundo@st.com
}

\begin{abstract}
The latest generation cars are often equipped with advanced driver assistance systems usually known as ADAS (Advanced Driver Assistance Systems). These systems are able to assist the car driver leveraging several levels of automation. It is therefore essential to adapt the ADAS technology to the car driver's identity in order to personalize the provided assistance services. For these reasons, such car driver profiling algorithms have been developed by scientific community. The algorithm herein proposed is able to recognize the driver's identity with an accuracy close to $99 \%$ thanks to ad'hoc specific analysis of the driver's PhotoPlethysmoGraphic (PPG) signal. In order to rightly identify the driver profile, the proposed approach uses a 1D Dilated Temporal Convolutional Neural Network architecture to learn the features of the collected driver's PPG signal. The proposed deep architecture is able to correlate the specific PPG features with subject identity enabling the car ADAS services associated to the recognized identity. Extensive validation and testing of the developed pipeline confirmed its reliability and effectiveness.
\end{abstract}

Keywords: ADAS · Deep learning · Automotive.

\section{Introduction}

The automotive industry is continually evolving to improve reliability and safety of the latest technology inside the cars. To meet this high demand for efficient and safe automotive systems, the technology is becoming increasingly sophisticated and the products currently on the market include intelligent solutions in the ADAS field [6, 19]. The ADAS systems, an acronym of Advanced Driver Assistance Systems, are becoming a very useful resource for the design of latest generation cars in order to increase the overall safety driving as well as to address classical automotive issues related to a drop of driver attention [18, 4]. Anyway, it is clear that the driving assistance systems must be customized to the driver and his driving dynamics. In particular. For this reason, it is necessary to profiling the driver or the recognition 
of his identity both before and during driving in order to enable the most appropriate ADAS assistance services. The present contribution is composed as follows. In the next section an examination of the prior art in relation to the driver profiling methods is presented. In the "Methods and Materials" section the proposed methodology will be illustrated in detail. Therefore in the "Results" section some performance indices of the proposed method will be shown which will be commented and discussed in the last section "Discussion and Conclusion".

\section{Related Works}

In [8], the authors analyzed several Android smartphone embedded sensors and classification pipelines in order to characterize the car driver behavior. The authors proposed a driver profiling approach by means of such Machine Learning based algorithms. More in detail, in the aforementioned survey [8] the authors have investigated several promising solutions based on the usage of Support Vector Machines (SVM), Random Forest (RF), Bayesian Network (BN) and Artificial Neural Networks (ANN). The final results confirmed that accelerometer and gyroscope represent the the most appropriate sensors to monitor the driving behaviour (also showing that the use of all sensor axes accomplish the task better than using a single one). In terms of machine learning architectures, they proved that the RF the best performing pipeline, followed by ANN even if the performance of both is satisfactory and equivalent, varying from 0.980 to 0.999 mean AUC values [8]. In [7] the authors proposed an interesting approach for profiling the car driver behaviour including identity recognition. In particular, trough a simulator they were able to analyse the keypressed dynamics of the analyzed subject. Through the analysis of the so collected key-pressed patterns, the authors were able to discriminate the identity of a specific driver with acceptable accuracy. In [11] a system named Driver Adaptive Vehicle Interaction System was implemented and analyzed. The main modules of the aforementioned method are the follows: the the Profile Management Module, the Driver Management Module and the Interaction Management Module. In particular, the first module is able to handle the car driver identity and correlated driver's driving characteristics. Through this collected data for each subject, the authors were able to provide a custom user-adaptive interaction system suitable to profile the driving dynamic. In [13] the authors performed a proper investigation about a model of human driving behaviour, and its correlated main issues. They described an interesting discussion about the principal human factors that might have an impact on driving: age, gender, personality, anger, mental stress, distraction, and so on. The authors have implemented and analyzed different pipelines with very interesting results. More details in [13]. In [5] the authors designed a pipeline named "Sense Fleet" based on the output analysis of such specific smartphone's sensors in order to identify and profile the subject who is driving. The method performs well but it suffers from the limitations of similar methods that use devices external to the car, therefore problems of invasiveness and compatibility with the automotive systems of the car. In [15] the author proposed an interesting car driver identity recognition based on the usage of combined approach which includes machine learning 
and dynamic time warping methodology. Through the analysis of such physiological signals of the car driver (collected from specific bio-sensor embedded on the car systems) the author was able to recognize the driver identity with high accuracy. The pipeline herein proposed is an improvement of the one described in [15]. Specifically, the authors propose an approach for the car driver identity recognition based on the analysis of the "physiological imprinting" of the subject [16]. The following section introduce and describe with more detail the proposed pipeline.

\section{Methods and materials}

In this section the overall proposed pipeline for the car driver identity recogntion will be described. The whole implemented pipeline is based on the use of an innovative analysis of the "physiological impriting" of the driver that the authors characterize through the photoplethysmographic signal (PPG) of the subject [16, 22]. The PPG signal represents a non-invasive physiological track of the subject's cardiovascular system correlated to the heart pulse-rate dynamic. This physiological signal, can be used to monitoring the heart pulse and respiratory rate of a subject [17]. Furthermore, the PPG signal may be also used to obtain a non-invasive measure the blood volume dynamic for several cardiovascular assessments [17,3]. A brief description of the PPG signal features is reported. A classical PPG waveform collected from a bio-sensor placed in contact with the skin of the examined subject contains a pulsatile ('AC') physiological waveform which is related to the cardiacsynchronous changes in the blood volume superposed with a slowly varying ('DC') component that contains the lower frequency information correlated to respiration, thermo-regulation [16]. The arteries and arterioles in the subcutaneous tissue of the analyzed subject's skin are stretched by the pressure of the blood pumped by the heart into the periphery, with a specific pressure, in each cardiac cycle (systolic phase). This dynamic tends to be reduced in the diastolic phase of cardiac activity $[17,3]$. Through ad-hoc designed bio-sensor it will be possible to capture the mentioned blood dynamics regulated by cardiac activity in order to reconstruct a signal that is strongly correlated with the aforementioned cardiac phases and therefore with the changes in blood flow. More in detail a small secondary peak, observed by a pressure pulse from the venous plexus, can be detected through a sensing device made-up by a light-emitter and photo-detector placed over the skin. The blood dynamic changes, will be detected by illuminating the skin and then by measuring the amount of light either transmitted or back-scattered to the coupled photo-detector. This is exactly the operating principle with which the PPG signal is constructed from a bio-sensor consisting of a light emitter and a photo-detector that captures the transmitted or back-scattered light. More detail in [16, 22, 17,3]. Therefore, the PPG signal can effectively be considered as a subject's "physiological imprint" or "cardiac imprint". The PPG waveform can be used as a fingerprint or bio-marker of a car driver; this implies that the features extracted from PPG signal can be properly used to recognize the subject from which the PPG is sampled [15]. In the Figure 1 the implemented PPG bio-sensor is reported. 


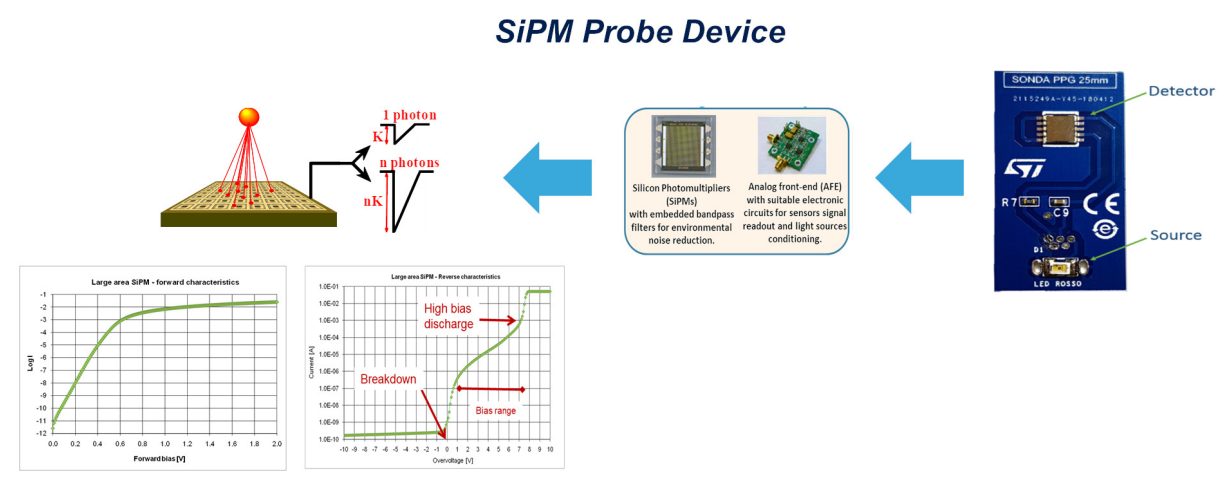

Fig. 1. The implemented PPG signal sensing probe.

The sensing device reported in Fig.1 and used by the authors for implementing the PPG sampling in the pipeline herein described, consists of two main components: a Silicon PhotoMultipliers (SiPMs) detector and two source LED emitters. The SiPM photo-detector used in our system is produced by STMicroelectronics (Catania, Italy) [25, 12]. The SiPM device has a total area of $4.0 \times 4.5 \mathrm{~mm}^{2}$ and 4871 square microcells with $60 \mu \mathrm{m}$ of pitch. It has a geometrical fill factor of $67.4 \%$ wrapped in a surface mount housing (SMD) of $5.1 \times 5.1 \mathrm{~mm}^{2}$ total area [25, 12, 24]. A Pixelteq dichroic bandpass filter (Bryan Dairy Rd, Largo, FL, USA) centered at $542 \mathrm{~nm}$ (Full Width at Half Maximum (FWHM) of $70 \mathrm{~nm}$ and optical transmission higher than $90 \%$ in the pass band range) was glued on the SMD package by using 352TM adhesive (Loctite ${ }^{\circledR}$, Milan, Italy). With the described setup, considering the driving range $0-3 V$, the device has a maximum Photon Detection Efficiency (PDE) of about $29.4 \%$ at $565 \mathrm{~nm}$ and of about $27.4 \%$ at $540 \mathrm{~nm}$ (central wavelength in the filter pass band). Moreover, a dichroic filter has been included in order to reduce the absorption of environmental light of more than $60 \%$ in the linear operation range. The emitter that has been used as optical light source is composed of two LT M673 LEDs (OSRAM, Milan, Italy). In particular, both LEDs are based on InGaN technology (in SMD package) emitting at $873 \mathrm{~nm}$, they also have an area of $2.3 \times 1.5 \mathrm{~mm}^{2}$ viewing angle of 120 and typical power emission of a few $\mathrm{mW}$ in the standard operation range. More details in [15-17]. In Fig. 1 the implemented sensing device is shown in which the part containing the SiPM photo-detector (Detector in Fig. 1) is highlighted, whose characteristic curves are shown. In addition, the part showing the LEDs (Source in Fig. 1) which in this case emit, as indicated, at a wavelength of $873 \mathrm{~nm}$, is highlighted. Introduced the bio-sensor to reconstruct the PPG signal, we now describe the implemented pipeline for the recognition of the driver's identity based on the processing of the physiological signals of the subject. An overall outline of the implemented car driver identity recognition pipeline is shown in Figure 2. Each block of designed pipeline will be described in the following subsections. 


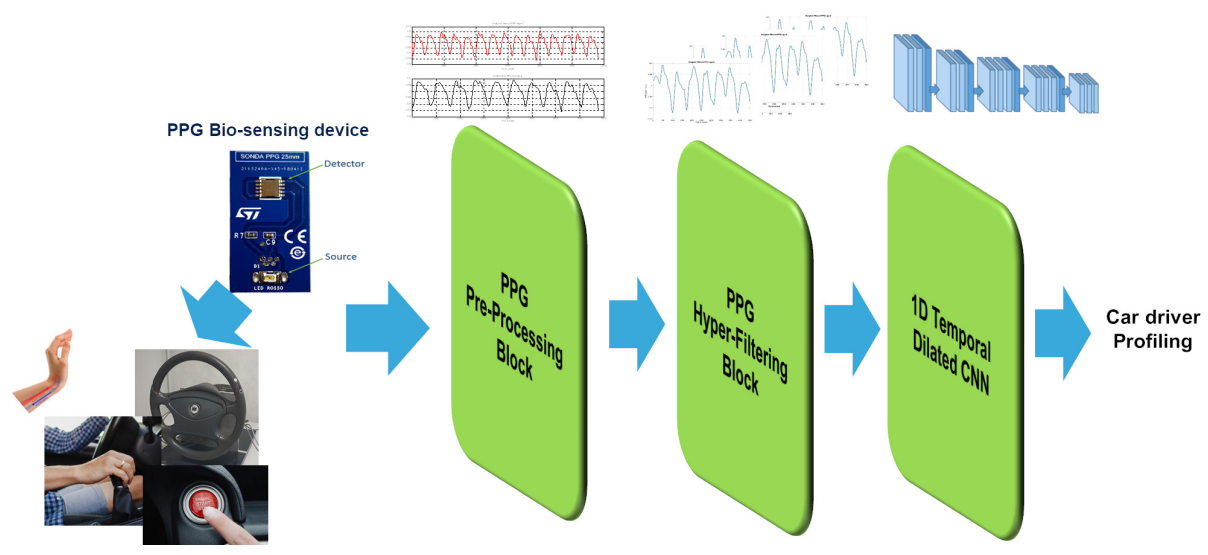

Fig. 2. Car driver identity recognition pipeline.

\subsection{The PPG Pre-Processing Block}

This block takes care of acquiring, filtering, stabilizing and pre-processing the PPG signal sampled from the driver. In order to be able to sample the so called physiological imprint of whom is driving the vehicle, the PPG sensing device will be embedde into different part of the car dashboard, specifically, in the car steering in the start button or in the gear shift. Each time the driver places the hand on the start button or on the steering or over the gear lever, the PPG signal will be sampled from the palm of the hand by means of the bio-sensing probes embedded in these parts of the car. In this way the PPG driver's signal will be persistently collected. The sensing devices so designed produce a raw PPG signal from the hand of the car driver. A microcontroller device detects the first raw PPG signal sampled by one of the biosensors placed in the various points of the car mentioned above and therefore selects the latter for the subsequent processing steps to recognize the driver's identity. To increase the robustness of the PPG sampling pipeline we have distributed several PPG sensors in the car's steering equidistant from each other as well as in the start button or in the gear shift. In the case where the driver will not put any hands on a PPG sensor placed in the car, the last identification setup will be considered. If no identity has been acquired when the vehicle is turned on, the system will asked to the driver to place the hand in one of the PPG sensors embedded in the steering wheel in order to proceed with identification. However, a recovery system based on innovative motion magnification algorithms (preliminary introduced in [23]]) is ongoing to be implemented for covering the issue related to the case in which the PPG signal is no longer available. More detail in the conclusion section of this paper. About the sampled raw PPG signal, as introduced, it is originally an optical signal which require to be converted before to be used in our pipeline. For this purpose, we used a 24-bits Analog to Digital Converters embedded in a board we designed for collecting and pre-processing the raw PPG signal. The Fig. 3, shows an instance of the designed motherboard which is integrated into the vehicle contro unit. More 
detail on the hardware setup implemented can be found in [25, 12, 24]. The PPG sensing devices located on the car's steering wheel/gear shift or start button have been plugged-in to the USB connectors of the developed motherboard. The so sampled raw PPG signal was managed by such algorithms running as firmware in a 32bits Micontrollers (STA1295 Accordo5 MCU) and SPCx Chorus MCU series [2, 1]. The so gathered raw PPG signal will be afterwards post-processed by the HyperFiltering block as described in the next section. The described hardware setup is reported in Figure 3.
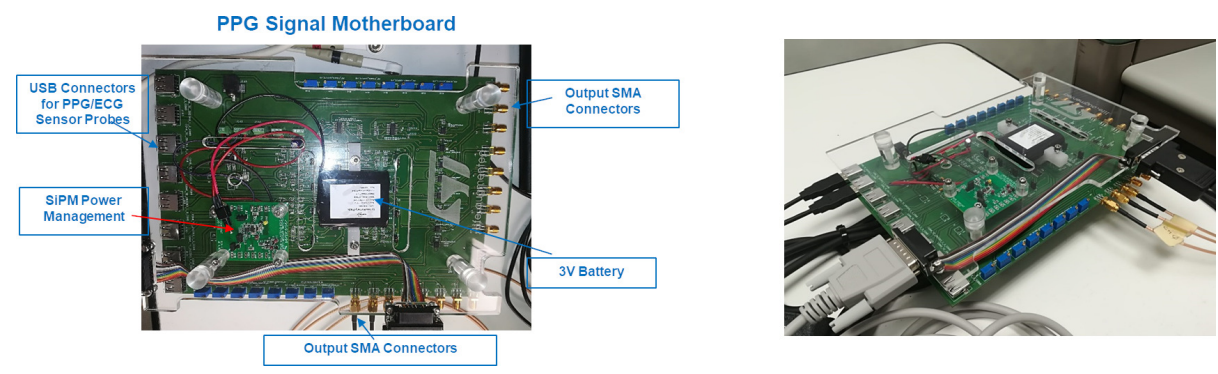

Fig. 3. The designed PPG signal pre'processing motherboard

\subsection{The PPG Hyper-Filtring Block}

To properly work with the PPG signal a frequency filter is needed since the raw format embeds several components useless for our goal. This particular detail have been carried out by the authors in [19][3, 25, 12, 24]. Furthermore, taking into account of a real driving scenario which includes motion artifacts, noises, car engine vibrations and so on, ad-hoc filtering or stabilization task is needed.Expressly, a classical approach for PPG filtering and stabilization is based on the usage of a set of FIR (Finitte Impulse Response) block designed to work band-pass filter in the range $0.5-10 \mathrm{~Hz}$. A classical FIR filter used for both low frequencies (low pass) and high frequencies (high pass) carry out a classic processing formalized by the following classic discrete-time equation:

$$
y_{P P G}[n]=\sum_{i=0}^{N_{0}} \delta_{i} \cdot x[n-i],
$$

where $N_{0}$ represents the order of the used filter, while the $\delta_{i}$ define the filter coefficients and $n$ the number of samples of the source raw PPG signal $x[k]$. Evidently, the signal $y_{P P G}[n]$ shows the filtered PPG waveforms. In the following table 1 the FIR setup usually applied for PPG filtering is reported [16].

In this contribution, the authors intend to propose a different solution in relation to the filtering of the raw $P P G$ signal. This idea will be better described in the 
Table 1. Low-pass and high-pass filter design for the photoplethysmgraphic (PPG) raw signal.

\begin{tabular}{ccccc}
\hline Type & $\begin{array}{c}\text { Frequency } \\
\text { pass }(\mathbf{H z})\end{array}$ & $\begin{array}{c}\text { Frequency } \\
\text { stop (Hz) }\end{array}$ & $\begin{array}{c}\text { Passband } \\
\text { Attenuation }(\mathbf{d B})\end{array}$ & $\begin{array}{c}\text { Stoband } \\
\text { Attenuation }(\mathbf{d B})\end{array}$ \\
\hline Low-pass & 3.8 & 7.21 & 0.001 & 100 \\
\hline High-pass & 1 & 0.3 & 0.01 & 40 \\
\hline
\end{tabular}

following paragraphs. In the pipeline described herein, we replace the above introduce classifica FIR filters setup with an-other set of properly configured hyperfiltering layers. The idea that we in-tend to propose in this work is inspired from the well known hyper-spectral method applied to 2D imaging [19]. Hyperspectral imaging, like other spectral imaging, collects visual information from across the whole electromagnetic spectrum. The goal of hyperspectral imaging methodology is to retrieve the so-called "frequency spectrum of each pixel" in order to address the classical image processing issues such as objects recognition, materials identification, and so on [19]. With aim to emulate the same approach, the authors investigated the effectiveness of applying the same approach to the study and analysis of 1D PPG signals. Specifically, the authors investigated whether, by collecting the information deriving from a "hyper-filtering" processing of the original 1D signal (PPG, in this case), we could obtain useful information to characterize the "frequency spectrum of each signal sample", that is, the information useful to address the problem for which the signal is analyzed in our case, the detection of the driver identity. For this reason, instead of applying a single filter set (low pass and high pass) we analyzed the source PPG signal over a range of frequencies that would allow us to better characterize the value of the single signal-sample. Considering that the useful frequency range, which allows to obtain an information component of the PPG signal, is included in the $0.5-10 \mathrm{~Hz}$ range, we have investigated the scenario of properly splitting this frequency range in sub-bands to be applied to simulate the phenomenon of hyper-filtering. Considering that, in the case of the PPG signal, it is necessary to apply both a low-pass and a high-pass filtering (therefore, a band-pass filter is required), we opted for two layers of hyper-filtering i.e. one that varied the frequencies in the low-pass band maintaining instead the cut-off frequency of the high-pass filter (hyper low-pass filtering layer) and, vice versa, one that varied the cut-off frequencies of the high-pass filter while maintaining constant the frequency of the low pass filter (hyper high-pass filtering layer). Furthermore, considering the need to have filters that do not create distortions in the PPG bandwidth, we decided to adopt the Butterworth filters in both layers of hyper-filtering [19]. As is known, Butterworth filters are the simplest electronic filters usable for signal processing applications [3, 25, 12, 24]. The first and most important part of the proposed method was related to the selection of the sub-bands intervals of the range $0.5-10 \mathrm{~Hz}$ and the relative cut-off frequencies. To address this issue, we have used a classical "trial and error" approach that would first search for the best number of sub-intervals in the $0.5-10 \mathrm{~Hz}$ frequency range. Specifically, after a series of heuristic tests, we selected a value equal to 11 sub-bands as the best trade-off between computational 
load and discriminative ability. Pratically, in our experiments, we found that when using a smaller number of frequency sub-bands $(<11)$, the detection performance driver identity decreased a lot. Meanwhile, increasing the number of sub-bands ( $>$ 11), the performances remained almost stable, although the computational load of the whole algorithm obviously increased. Therefore, in order to find a correct trade-off between performance and computational load, we established that 11 sub-bands were enough to properly discriminate the driver identity. Therefore, for each hyper-filtering layer, we proceeded to split the range of applicable frequency in 11 specific sub-bands. Once the number of sub-bands was set, we put together a reinforcement learning algorithm structured as follows:

- We defined an action $a_{t}$ as the sub-band frequency value between $0.5 \mathrm{~Hz}$ and cut-off frequency according to the type of filtering (low-pass or high-pass);

- We defined an Agent select the action $a_{t}$;

- We defined a next state $S_{t+1}$ as a set of pre-processed signals obtained collecting the value of each input PPG samples (in a windows of 5 sec sampling at 1 $\mathrm{KHz}$ as sampling frequency) of the filtered PPG raw signal at specific sub-band frequency of the action $a_{t}$;

- We define an environment Reward as $R\left(. \mid s_{t}, a_{t}\right)$, that is, a measure of drowsiness of the car driver. We defined as $R\left(. \mid s_{t}, a_{t}\right)$ the distance of the output of ad-hoc machine learning system (regression layer plus SoftMax classification) with respect to the actual driver identity;

We are interested to determine the optimal policy $P_{o}$ that minimizes the cumulative discount reward:

$$
P_{o}=\operatorname{argmax}_{P_{o}} E\left[\sum_{t \geq 0} \gamma^{t} R\left(. \mid s_{t}, a_{t}\right) \mid P_{o}\right],
$$

where $\gamma$ is a proper discounted coefficient in $(0,1)$. In order to evaluate the the goodness of a state $s_{t}$ and the goodness of a state-action couple $\left(s_{t}, a_{t}\right)$, we defined the value function and the $\mathrm{Q}$-value function, respectively, as follows:

$$
\begin{gathered}
V^{P_{0}}\left(s_{t}\right)=E\left[\sum_{t \geq 0} \gamma^{t} R\left(. \mid s_{t}\right) \mid P_{o}\right] \\
Q^{P_{0}}\left(s_{t}, a_{t}\right)=E\left[\sum_{t \geq 0} \gamma^{t} R\left(. \mid s_{t}, a_{t}\right) \mid P_{o}\right] .
\end{gathered}
$$

By solving the above models through classical Q-learning algorithms [19], we found the following set of sub-band frequency for each hyper-filtering layer. Once we have identified the optimal frequency setup using the described RL algorithm , the latter system will be disconnected from the main pipeline as it is no longer needed for the operation of the proposed approach concerning the discrimination of the driver identity. Therefore, the RL algorithm is needed simply as "one-shot" optimization algorithm to be used only in the initial setup of the filtering frequency framework, and thus it will no longer needed for achieving the target of the proposed solution. Once the frequency sets of the two hyper-filtering filter sub-systems 
have been identified, the sampled raw PPG signal will be processed accordingly. Formally, if we set with $x(k)$ the sampled raw PPG signal, for each frequency setup, we obtain the following hyper-filtered time series:

$$
\begin{aligned}
& \varphi_{H_{L P}}^{i}(k)=F_{B u t t e r w o r t h}\left(f_{L}^{i}, f_{H}, x(k)\right) i=1,2, \ldots 11 ; k=1,2, \ldots . . n, \\
& \varphi_{H_{H P}}^{i}(k)=F_{\text {Butterworth }}\left(f_{L}, f_{H}^{i}, x(k)\right) i=1,2, \ldots 11 ; k=1,2, \ldots n,
\end{aligned}
$$

where $\varphi_{H_{H P}}^{i}(k)$ and $\varphi_{H_{L P}}^{i}(k)$ represent the set of hyper-filtered time series coming from hyper-filtering high-pass and low-pass processing, respectively. The function $\mathbf{F}_{\text {Butterworth }}$ represents the filter processing performed by the so configured Butterworth filter, while $f_{L}, f_{H}$ represent the fixed cut-off frequencies and $f_{H}^{i} f_{L}^{i}$ the variable frequency (fc-pass-x) as per Tables 2 and 3 . Now, for each sample of the single so hyper-filtered PPG signals, a dataset of further signals will be created, each having a temporal dynamics represented by the intensity value of the single sample of the so filtered PPG signal.

Table 2. Hyper Low-Pass Filtering Setup (in Hz)

\begin{tabular}{|c|c|c|c|c|c|c|c|c|c|c|c|}
\hline F & Fc-pass-1 & Fc-pass-2 & Fc-pass-3 & Fc-pass-4 & Fc-pass-5 & Fc-pass-6 & Fc-pass-7 & Fc-pass-8 & Fc-pass-9 & Fc-pass-10 & Fc-pass-11 \\
\hline HP & 0.5 & $/$ & $/$ & $/$ & $/$ & $/$ & $/$ & $/$ & $/$ & $/$ & $/$ \\
\hline LP & 1.00 & 1.34 & 2.09 & 2.231 & 3.09 & 3.44 & 4.2 & 4.23 & 5.2 & 5.52 & 6.87 \\
\hline
\end{tabular}

Table 3. Hyper High-Pass Filtering Setup (in Hz)

\begin{tabular}{|c|c|c|c|c|c|c|c|c|c|c|c|}
\hline F & Fc-pass-1 & Fc-pass-2 & Fc-pass-3 & Fc-pass-4 & Fc-pass-5 & Fc-pass-6 & Fc-pass-7 & Fc-pass-8 & Fc-pass-9 & Fc-pass-10 & Fc-pass-11 \\
\hline HP & 0.5 & 1.5 & 2.2 & 2.75 & 3.12 & 3.65 & 4.1 & 4.48 & 5.23 & 5.3 & 6.11 \\
\hline LP & 7 & $/$ & $/$ & $/$ & $/$ & $/$ & $/$ & $/$ & $/$ & $/$ & $/$ \\
\hline
\end{tabular}

Formally, if we indicate with $W_{P P G}^{i}\left(t_{k}\right)$ the single segmented waveform of each hyper-filtered PPG time series, we proceed computing for each sample $s(t k)$ of the waveform $W_{P P G}^{i}\left(t_{k}\right)$ a signal-pattern depending on how that sample $s(t k)$ varies in intensity in the various hyper-filtered PPG signals $\varphi_{H_{H P}}^{i}(k)$ and $\varphi_{H_{L P}}^{i}(k)$ for $i=$ $1,2, \ldots, 11$. In this way, we will obtain a fairly large dataset of signals whose length will thus be equal exactly to the number of filtering frequencies, that is, 11 in this application setup. The following equations show how we obtain the signal-patterns $\zeta_{H P}^{k}\left(s\left(t_{k}\right)\right)$ and $\zeta_{L P}^{k}\left(s\left(t_{k}\right)\right)$ for each sample of the acquired PPG waveforms:

$$
\zeta_{H P}^{k}\left(s\left(t_{k}\right)\right)=\left[\varphi_{H_{H P}}^{1}\left(t_{k}\right), \varphi_{H_{H P}}^{2}\left(t_{k}\right), \ldots \varphi_{H_{H P}}^{11}\left(t_{k}\right)\right] k=1,2, \ldots n,
$$




$$
\zeta_{L P}^{k}\left(s\left(t_{k}\right)\right)=\left[\varphi_{H_{L P}}^{1}\left(t_{k}\right), \varphi_{H_{L P}}^{2}\left(t_{k}\right), \ldots \varphi_{H_{L P}}^{11}\left(t_{k}\right)\right] k=1,2, \ldots n
$$

The following Fig. 4 show some instances of the hyper-filtering PPG time se$\operatorname{ries} \varphi_{H_{H P}}^{i}(k)$ and $\varphi_{H_{L P}}^{i}(k)$, as well as related generated signal patterns $\zeta_{H P}^{k}\left(s\left(t_{k}\right)\right)$ and $\zeta_{L P}^{k}\left(s\left(t_{k}\right)\right)$. As for the classic hyper-spectral method, by means of the hyper-filtering approach herein described we are able to highlight multiple features of the PPG source signal as the frequency setup varies so as to significantly increase the discriminating features relating to the source sampled PPG signal of each subject. The dynamic of the signal-patterns $\zeta_{H P}^{k}\left(s\left(t_{k}\right)\right)$ and $\zeta_{L P}^{k}\left(s\left(t_{k}\right)\right)$ shows how the single sample of the acquired PPG time series varies according to the applied frequency filter setup. We collected several signal-patterns as we performed the analysis of each sample of the hyper-filtered PPG time series in a proper timing window after the car-driver put the hand over the steering wheel or in other part in which we have embedded the PPG sensor probe. The generated signal-patterns will be fed as input of the designed deep classifier block, as described in the following sub-section.
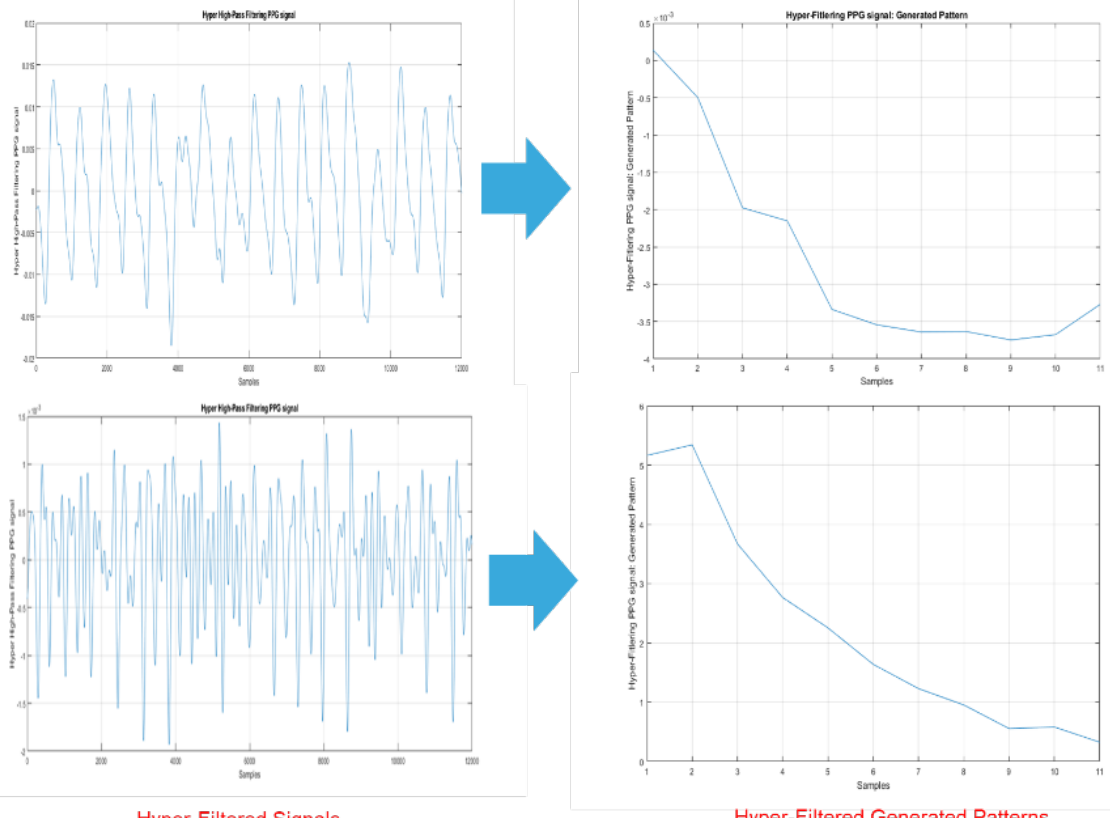

Hyper-Filtered Generated Patterns

Fig. 4. In the left column some instances of the hyper-filtered high-pass PPG time series $\varphi_{H_{H P}}^{i}(k)$; In the right column an instance of the corresponding generated signal-patterns: $\zeta_{H P}^{k}\left(s\left(t_{k}\right)\right) ;(\mathrm{c}),(\mathrm{d}) \zeta_{L P}^{k}\left(s\left(t_{k}\right)\right)$. 


\subsection{The 1D Temporal Dilated Deep Classifier}

Ad-hoc 1D Temporal Dilated Convolutional Neural Network (1D-CNN), has been designed for classifying the collected hyper-filtered PPG signal patterns [21]. The following Figure 5 report the overall scheme of the proposed 1D deep classifier.

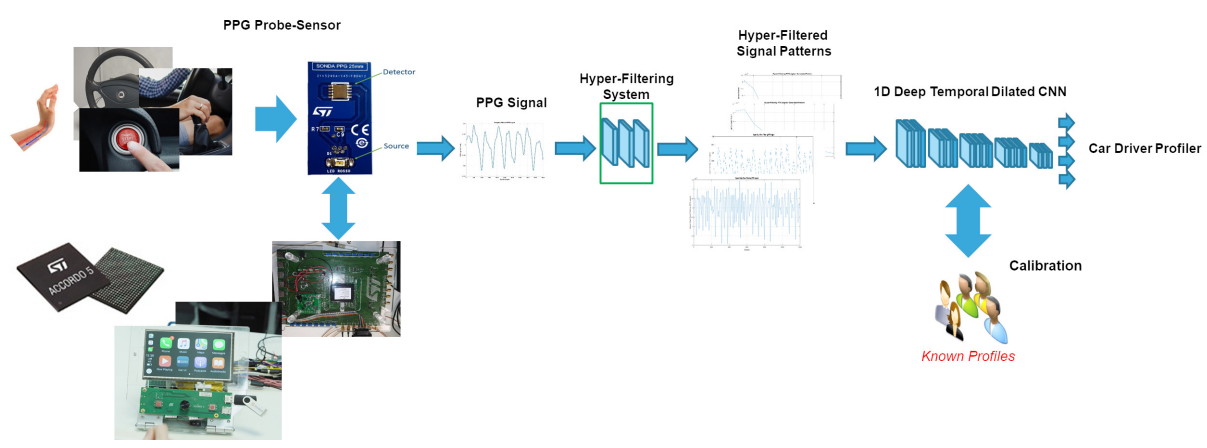

Fig. 5. The 1D Deep Classifier based pipeline for a robust car driver physiological identification

Our proposed network takes as input the signal-patterns $s\left(t_{k}\right)$ generated from each PPG hyper-filtered signals. The main novelty of the proposed architecture is the introduction of dilated causal convolution layers. The term "causal" denotes that the activation at time $t$ depend on the activation computed at time $t-1$. The proposed 1D-CNN includes such multiple residual blocks. Specifically, the proposed deep network consists of a sequence of 16 residual blocks stacked together. Each block consists of a dilated convolution layer, batch normalization, ReLU and a spatial dropout. A dilated layer includes a $3 \times 3$ convolution operation. The dilation factor is set to 2 increasing for each block up to the eighth block. After no dilation it is applied. A downstream softmax layer completes the proposed pipeline. The output of the designed 1D-CNN predicts the driver identity from source hyper-filtered PPG generated signal-patterns. Specifically, the output of the 1D-CNN, thus, is able to predict the specific driver profile or identity which turns out to be the most likely profile among those on which it has been earlier trained by means of a system calibration phase. Consequently, the profiles of users who are allowed to obtain specific services enabled by the car control unit. In fact, a preliminary calibrationtraining phase of the algorithm allows this to be trained on specific profiles related to very exact identities of drivers. For each of the recruited drivers for the training phase, the PPG signal is acquired through the described pipeline and then the hyper-filtering block is applied in order to obtain the features to train the deep classifier so that it associates the retrieved features with the driver identity from which the PPG signal is sampled. So, as soon as a subject start to drive the car and the related PPG signal is sampled by the bio-sensors placed in the car's passenger compartment, according to the so sampled PPG signal and its related patterns obtained from the Hyper-filtering system, the classifier will be able to output a con- 
fidence level for each profile previously learned in the training-calibration phase. The above-mentioned confidence level, in our case, represents the percentage of belonging of the patterns to the previously learned driver profiles. If a new driver is driving whose identity does not match to any learned profiles in the calibration phase, a low probability (under 50\%) will output by the classifier for each confidence level. The results, detailed in the next section, confirms that the implemented Deep Learning framework is able to identify the right car driver profile with high accuracy. The implementd 1D Deep CNN backbone is ongoing to be ported over the STA1295 Accordo5 embedded MCU platform with Linux YOCTO as Opertaing System $[2,1]$. Fig. 6 shows a loss dynamic of the 1D-CNN properly trained.

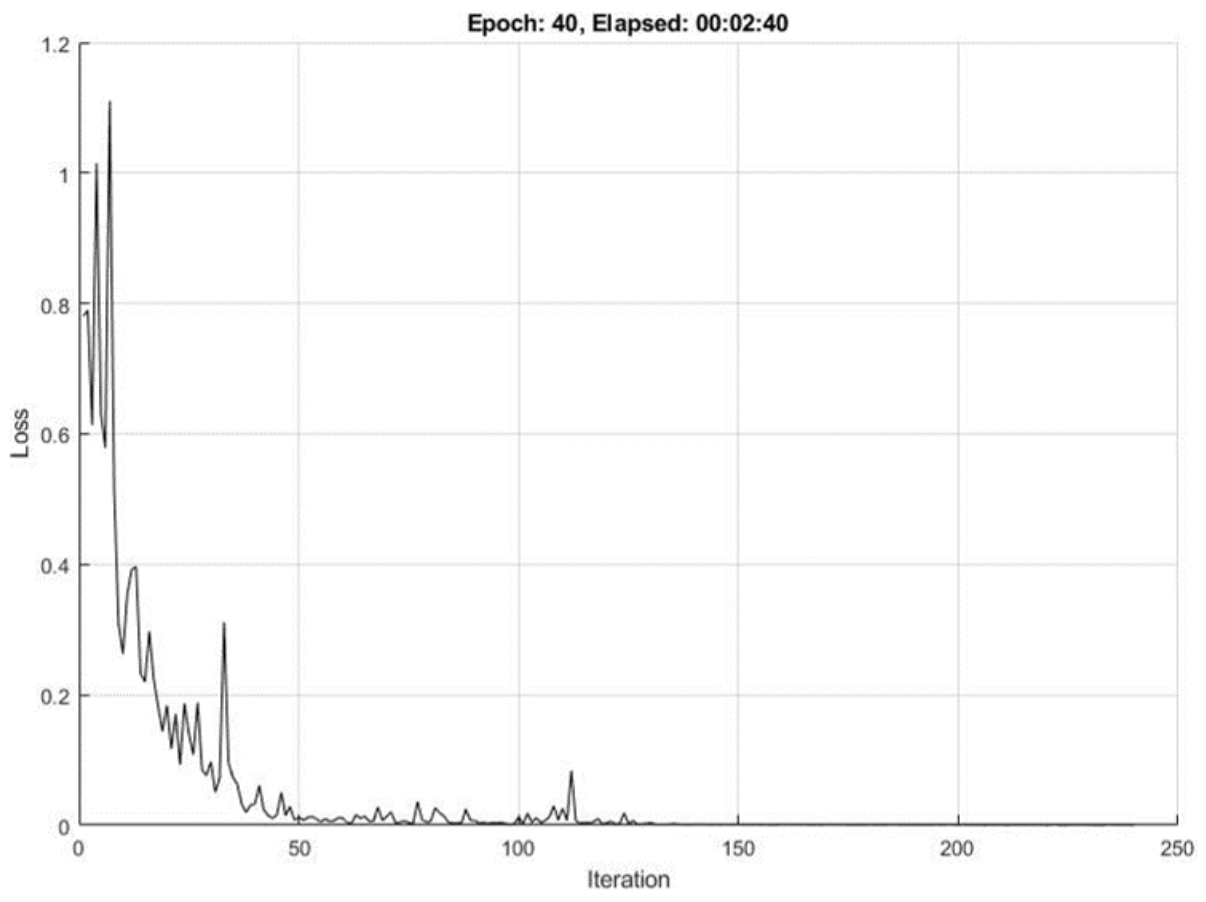

Fig. 6. The loss dynamic of the 1D-CNN.

\section{Results}

In order to validate the proposed pipeline, the authors asked for support from a team of physiologists they are with were collected several PPG measurements of different subjects. In order to have a complete mapping of the PPG signal dynamic of each recruited subject, the physiologists stimulated several physiological states (inattentive, enthusiastic, stressed, and so on) monitoring the subject through the 
ElectroEncephaloGraphic (EEG). Experiments were performed on 75 mixed healthy subjects: 35 men and 40 women aged between 20 and 79 years; none of the participants were using drugs suitable of changing cortical excitability. Volunteers authorised informed consent to the procedures approved by the Ethical Committee Catania 1 (authorization n.113 / 2018 / PO) in according to the Declaration of Helsinki. Participation criteria encompassed the possession of a valid driving licence for motor vehicles. Our team have collected 10 minutes of PPG signal with the bio-sensing device described in previous sections and based on coupled LED-SiPM technology with sampling frequency of $1 \mathrm{kHz}$. A car has been equipped with six LED-SiPM coupled sensing device placed equidistant from each other in order to cover the most common driving styles of a subject. The implemented pipeline was hosted by the following setup: MATLAB full toolboxes vers. $2019 \mathrm{~b}$ running in a server having an Intel 16-Cores and NVIDIA GeForce RTX 2080 GPU. We have configured a scenario of 4 known car driver profiles to be identified by proposed pipeline. The scenario included two young and two aged people from opposite sex. The subjects were chosen from the dataset described above and there was not any relationship between them. All other subjects are recognised as unknown profiles. To be able to test the designed pipeline, the acquired PPG data for the selected known profiles have been divided as follow: $70 \%$ has been used for training and validation while the remaining $30 \%$ for testing. In order to verify both in the training phase and in the testing phase if the algorithm was able to recognise the identity of the drivers, the authors have constructed artificial PPGs composed of subset of waveforms coming from various recruited subjects (both from the selected four known subjects as well as the remaining unknown subjects). The overall accuracy (both training and test) of the described method stands at around $98.75 \%$, thus, confirming the robustness of the pipeline proposed in this contribution. The previous pipeline [15] tested in this recruited dataset with four different identity to be recognized showed a performance of $97.12 \%$ significantly lower than the method proposed here. Although it is a matter of a few percentage points of difference between the two pipelines, in the case of automotive applications, the accuracy must be as high as possible.

\section{Discussion and Conclusion}

The aforementioned experimental results confirm the ability of the proposed pipeline to recognise the car driver identity by analysing some physiological features of related PPG signal. The suggested approach has introduced the concept of "cardiac imprinting or "physiological imprinting" as a valuable replacement of the classic fingerprint to outline and correctly identify a subject. Specifically, through the designed contribution the embedded ADAS system will be able to consistently monitor the driver's identity, steadily knowing whom is driving. Moreover, by recognition of the driver identity, the mentioned approach will be able to offer congruous information to the car control unit for enabling the services and ad-hoc configurations for the recognized identity, providing a tailor-made ADAS systems. The performance accuracy confirmed the effectiveness of the proposed approach. As future works of the presented work, the authors are researching the integration of vision 
algorithms able to analyze also the anxiety and stress level of the driver, in order to improve the driving safety and assistance systems. In conclusion, preparatory and encouraging results are being found by examining the driver's identity recognition within the fusion of data coming from both (PPG) physiological signals. A promising results were obtained by integrating some statistical analyzes (Markov models) of the integrated driver's PPG signal with some visual features extracted from the viewer's face acquired from a camera located on the dashboard [21, 20, 9]. Future scientific contributions will show these results which also integrate the use of Deep Long Short Term Memory and further Reinforment Learning model for the characterization of discriminating features $[14,10]$.

\section{References}

1. STMicroelectronics ACCORDO 5 Automotive Microcontroller, https://www.st.com/en/automotive-infotainment-and-telematics/automotiveinfotainment-socs.html?icmp=tt4379_gl_pron_nov2016, (accessed on 20 Feb 2020)

2. STMicroelectronics SPC5 MCUs, https://www.st.com/en/automotivemicrocontrollers/spc5-32-bit-automotive-mcus.html, (accessed on 20 Feb 2020)

3. Banna, G.L., Camerini, A., Bronte, G., Anile, G., Addeo, A., Rundo, F., Zanghi, G., Lal, R., Libra, M.: Oral metronomic vinorelbine in advanced non-small cell lung cancer patients unfit for chemotherapy. Anticancer research 38(6), 3689-3697 (2018)

4. Caber, N., Langdon, P.M., Clarkson, P.J.: Intelligent driver profiling system for cars-a basic concept. In: International Conference on Universal Access in Human-Computer Interaction. pp. 201-213. Springer (2018)

5. Castignani, G., Frank, R.: SenseFleet: A smartphone-based driver profiling platform. In: 2014 Eleventh Annual IEEE International Conference on Sensing, Communication, and Networking (SECON). pp. 144-145. IEEE (2014)

6. Conoci, S., Rundo, F., Fallica, G., Lena, D., Buraioli, I., Demarchi, D.: Live demonstration of portable systems based on silicon sensors for the monitoring of physiological parameters of driver drowsiness and pulse wave velocity. In: 2018 IEEE Biomedical Circuits and Systems Conference (BioCAS). pp. 1-3. IEEE (2018)

7. Dangra, B.S., Rajput, D., Bedekar, M., Panicker, S.S.: Profiling of automobile drivers using car games. In: 2015 International Conference on Pervasive Computing (ICPC). pp. 1-5. IEEE (2015)

8. Ferreira, J., Carvalho, E., Ferreira, B.V., de Souza, C., Suhara, Y., Pentland, A., Pessin, G.: Driver behavior profiling: An investigation with different smartphone sensors and machine learning. PLoS one 12(4), e0174959 (2017)

9. Grasso, G., Perconti, P., Plebe, A.: Assessing social driving behavior. In: International Conference on Intelligent Human Systems Integration. pp. 111-115. Springer (2019)

10. Grasso, G.M., Lucifora, C., Perconti, P., Plebe, A.: Evaluating mentalization during driving. In: VEHITS. pp. 536-541 (2019)

11. Kim, K., Choi, H., Jang, B.: Design of the Driver-Adaptive Vehicle Interaction System. In: 2018 International Conference on Information and Communication Technology Convergence (ICTC). pp. 297-299. IEEE (2018)

12. Mazzillo, M., Maddiona, L., Rundo, F., Sciuto, A., Libertino, S., Lombardo, S., Fallica, G.: Characterization of sipms with nir long-pass interferential and plastic filters. IEEE Photonics Journal 10(3), 1-12 (2018) 
13. Mubasher, M.M., Jaffry, S.W., Jahangir, R.: Modeling of individual differences in carfollowing behaviour of drivers. In: 2017 International Multi-topic Conference (INMIC). pp. 1-7. IEEE (2017)

14. Rundo, F:: Deep LSTM with Reinforcement Learning Layer for Financial Trend Prediction in FX High Frequency Trading Systems. Applied Sciences 9(20), 4460 (2019)

15. Rundo, F.: Deep lstm with dynamic time warping processing framework: A novel advanced algorithm with biosensor system for an efficient car-driver recognition. Electronics 9(4), 616 (2020)

16. Rundo, F., Conoci, S., Ortis, A., Battiato, S.: An advanced bio-inspired photoplethysmography (PPG) and ECG pattern recognition system for medical assessment. Sensors 18(2), 405 (2018)

17. Rundo, F., Petralia, S., Fallica, G., Conoci, S.: A nonlinear pattern recognition pipeline for PPG/ECG medical assessments. In: Convegno Nazionale Sensori. pp. 473-480. Springer (2018)

18. Rundo, F., Rinella, S., Massimino, S., Coco, M., Fallica, G., Parenti, R., Conoci, S., Perciavalle, V.: An innovative deep learning algorithm for drowsiness detection from EEG signal. Computation 7(1), 13 (2019)

19. Rundo, F., Spampinato, C., Conoci, S.: Ad-hoc shallow neural network to learn hyper filtered photoplethysmographic (ppg) signal for efficient car-driver drowsiness monitoring. Electronics 8(8), 890 (2019)

20. Rundo, F., Trenta, F., Di Stallo, A.L., Battiato, S.: Advanced Markov-based machine learning framework for making adaptive trading system. Computation 7(1), 4 (2019)

21. Rundo, F., Trenta, F., di Stallo, A.L., Battiato, S.: Grid trading system robot (GTSbot): A novel mathematical algorithm for trading FX market. Applied Sciences 9(9), 1796 (2019)

22. Taeihagh, A., Lim, H.S.M.: Governing autonomous vehicles: emerging responses for safety, liability, privacy, cybersecurity, and industry risks. Transport Reviews 39(1), 103128 (2019)

23. Trenta, F., Conoci, S., Rundo, F., Battiato, S.: Advanced motion-tracking system with multi-layers deep learning framework for innovative car-driver drowsiness monitoring. In: 2019 14th IEEE International Conference on Automatic Face \& Gesture Recognition (FG 2019). pp. 1-5. IEEE (2019)

24. Vinciguerra, V., Ambra, E., Maddiona, L., Oliveri, S., Romeo, M.F., Mazzillo, M., Rundo, F., Fallica, G.: Progresses towards a processing pipeline in photoplethysmogram (PPG) based on SiPMs. In: 2017 European Conference on Circuit Theory and Design (ECCTD). pp. 1-5. IEEE (2017)

25. Vinciguerra, V., Ambra, E., Maddiona, L., Romeo, M., Mazzillo, M., Rundo, F., Fallica, G., di Pompeo, F., Chiarelli, A.M., Zappasodi, F., et al.: PPG/ECG multisite combo system based on SiPM technology. In: Convegno Nazionale Sensori. pp. 353-360. Springer (2018) 